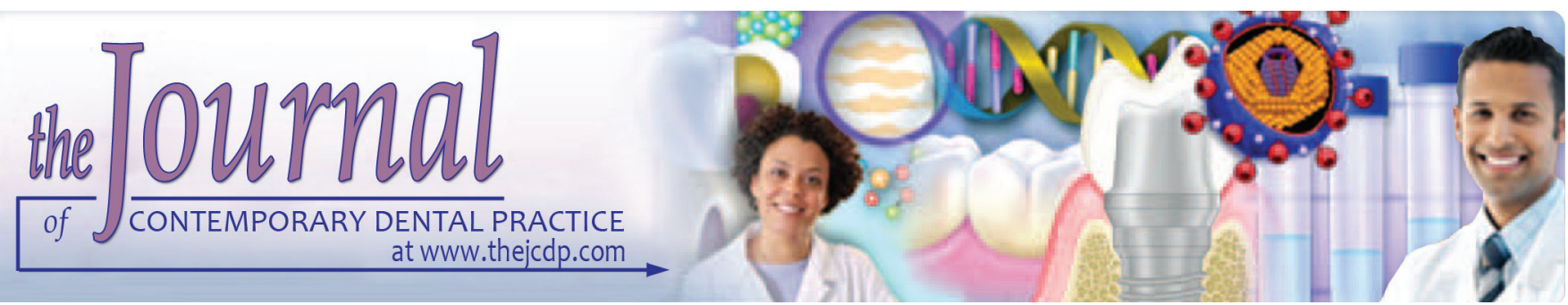

\title{
Prosthetic Rehabilitation using a Telescopic Crown constructed with Computer-assisted Design and Computer-assisted Manufacturing: A Case Report with a 2-year Follow-up
}

\author{
Nasser M Al Ahmari
}

\begin{abstract}
Aim and background: This case report describes a 74-year-old female patient whose teeth were rehabilitated using telescopic crowns (TCs) on abutments and fixed dental prostheses (FDPs) in the maxillary arch with the aid of computer-assisted design and computer-assisted manufacturing (CAD/CAM) technology. The mandibular arch was restored using casted removable dental prostheses (RDPs) and surveyed crowns.
\end{abstract}

Case report: The treatment sequences were followed as recommended in the dental literature. First, existing defective restorations were eliminated, caries was removed, and excellent mouth preparation was performed. Subsequently, root canal treatments and post and core placements were conducted. The casts were mounted, and a new vertical dimension was established. Maxillary telescopic FDP and mandibular prostheses were fabricated. Finally, a regular and proper follow-up and maintenance program was implemented.

Conclusion: The patient was satisfied with the esthetics and function of the telescopic FDP in the presence of the established occlusion.

Clinical significance: The secondary coping of the telescopic FDP is cemented with temporary cement, which can be changed easily whenever it is loose. Furthermore, it is completely opposed to RDP in the mandibular posterior masticatory area. The prognoses will be good improvement; the patient was medically fit, motivated, and followed continual oral hygiene protocol.

Keywords: Computer-assisted design, Computer-assisted manufacturing, Esthetic, Maxilla, Telescopic crown.

Department of Prosthetic Dental, King Faisal Medical City, Abha Kingdom of Saudi Arabia

Corresponding Author: Nasser M Al Ahmari, Department of Prosthetic Dental, King Faisal Medical City, Abha, Kingdom of Saudi Arabia, e-mail: nmr.dnt@gmail.com
How to cite this article: Al Ahmari NM. Prosthetic Rehabilitation using a Telescopic Crown constructed with Computer-assisted Design and Computer-assisted Manufacturing: ACase Report with a 2-year Follow-up. J Contemp Dent Pract 2018;19(4):468-474.

Source of support: Nil

Conflict of interest: None

\section{INTRODUCTION}

Prosthetic rehabilitation of an elderly partially edentulous patient can be performed using a wide range of different treatment options. The most preferred prosthetic materials are tooth or implant-supported FDPs, RDPs, and tooth/implant-supported overdentures in sequence. ${ }^{1}$

Telescopic crowns are also known as a double crown or crown-and-sleeve coping. These crowns were introduced at the beginning of the 20th century as retainers for RDPs. The TC consists of an inner (primary) telescopic coping that is permanently cemented to an abutment and a congruent detachable outer (secondary) TC that is rigidly connected to a detachable prosthesis. The inner parts or copings protect the underlying abutment from dental caries and retain and stabilize the secondary crowns. The secondary crown is engaged to the primary coping to form a telescopic unit, and it serves as an anchor for the remaining prostheses. ${ }^{2,3}$ These crowns, either prefabricated or postfabricated, are indicated in an unattainable draw path, limited finances, contraindication for surgery, and weak distal abutment that should not be used in FDP because of questionable prognosis. These TCs aid in the transfer of forces along the direction of the long axis in the abutment teeth and provide guidance, support, and protection from movement that may dislodge the FDPs or RDPs. ${ }^{4-6}$ 
The CAD/CAM technology is currently available and can be used to fabricate prostheses from single unit to complex full-arch constructions predictably. The CAD/ CAM technology can also be planned virtually and milled in a parallel path with a precise and passive fit in each dental arch. ${ }^{7}$

The stability and retention of the telescopic FDP are directly related to the number and distribution of abutments in the dental arch and the total convergence angle of the primary coping. A high frictional retention of the retainer is obtained from a smaller taper degree. The abutment walls with short clinical height should be kept parallel to each other, or the taper degree of the wall should be reduced $\left(2-5^{\circ}\right)$ to improve the retention. ${ }^{8}$ This clinical report describes the prosthetic rehabilitation of an old female patient with telescopic FDPs in the maxillary arch opposing a removable partial dental prosthesis with surveyed crown.

\section{CASE REPORT}

A 78-year-old female patient was referred for a prosthodontic evaluation and treatment from her dentist. Her main complaint was her upper anterior teeth. The patient was medically fit. She had undergone multiple extractions, FDPs, and RPDs many years ago. Her teeth \#12 and \#22 were also extracted 1 year ago. She does not visit her dentist regularly, does not brush her teeth on a regular daily basis, but uses Miswak two times a day.

Additional oral examination showed that the interpupillary and intercommissure lines were almost parallel at rest position and while smiling. Intraoral examination of the palate, mouth floor, tongue, buccal mucosa, and oropharynx showed no abnormalities. The maxillary arch was U-shaped. Existing metal-ceramic (MC) bridges extended from tooth \#17 to \#14 and from tooth \#23 to \#26. Tooth \#27 was restored with amalgam. The maxillary central incisors were prepared previously with no crowns. Moreover, the existing dentition with defective restorations, localized gingival inflammations, and generalized wear facets were noted (Figs 1A to C). The mandibular arch was U-shaped with missing bilateral posterior teeth. Dental caries was detected on teeth \#42 and \#43. The periodontal finding showed poor oral hygiene and recession on all existing teeth (Figs 1B and C).

The pretreatment panoramic radiograph showed finely woven and dense trabecular bone pattern. In general, mild-to-moderate horizontal bone loss was detected (Fig. 2A). Abutments in the maxillary sextants exhibited an insufficient tooth structure and required adjunctive therapy, i.e., periodontal and/or endodontic procedures. Reestablishment of the entire occlusal scheme was required with a change in the existing vertical dimension of occlusion (VDO). Complete mouth survey radiographs showed that the maxillary and mandibular teeth were partially dentate with generalized chronic moderate periodontitis. Multiple missing teeth, defective restorations, dental caries on several teeth, and an acute apical abscess in tooth \#26 were observed (Fig. 2B).

A diagnostic cast was made using irreversible hydrocolloid and dental stone. The face-bow transfer was
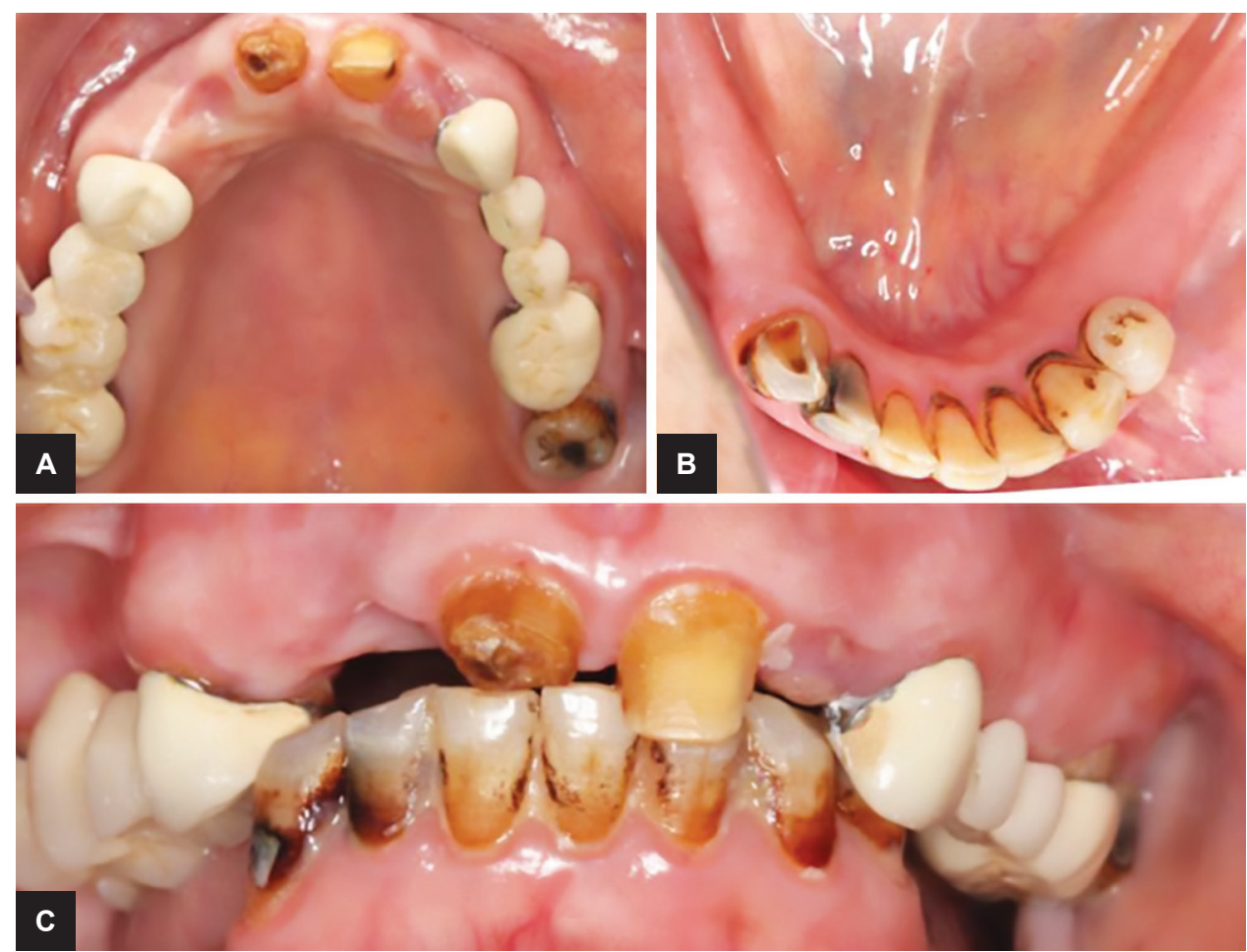

Figs 1A to C: Preoperative intraoral view 

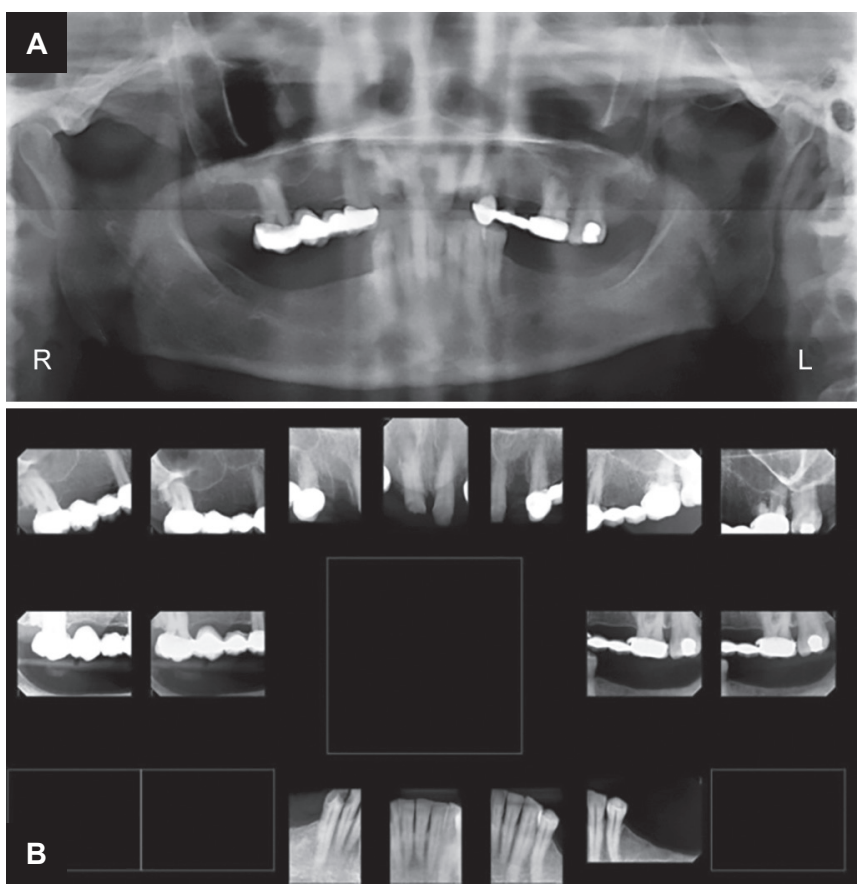

Figs 2A and B: Preoperative radiographs

utilized to mount the maxillary cast. The initial centric relation record was obtained to mount the study casts and verified using the split cast technique and intraoral observation. The occlusion analysis revealed an insignificant discrepancy between the centric occlusion and maximum intercuspation at the existing VDO. The first repeatable contact was between tooth \#23 and teeth \#34 and \#33 with no right mediotrusive contacts. The right laterotrusive movement was guided by tooth \#14 over \#43 with no left mediotrusive contacts.

After the collected data were interpreted, and the oral examination was conducted, the casts were mounted on a Denar Mark II Articulator (Whip Mix Corp., Louisville, KY) with diagnostic wax-up at the existing occlusal vertical dimension. Appropriate occlusal planes and scheme were reestablished (Fig. 3). The treatment plan, which followed the phases suggested by Rosenstiel et al, ${ }^{9}$ was explained to the patient.

In Phase I, a multidisciplinary consultation was conducted. Deep scaling with root planing was performed, and the patient was instructed for oral hygiene measures. All defective restorations were removed, and caries control was conducted on all remaining teeth. Finally, Phase I was reevaluated.

In Phase II, endodontic treatments were carried out on affected teeth. Subsequently, casted posts (DuraLay, Reliance Dental Manufacturing Company, Italy) were fabricated and cemented on teeth \#11, \#26, \#42, and \#43. All teeth were prepared using vacuum-formed clear matrices as guide. Provisional restorations (Success SD, PROMEDICA, Neumunster, Germany) were directly

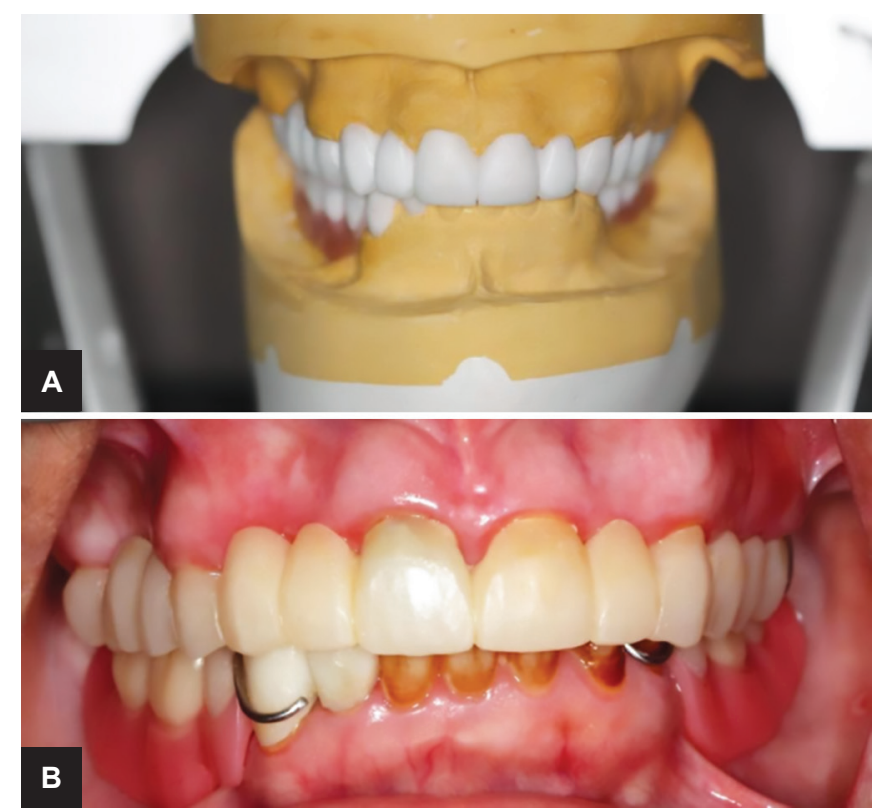

Figs 3 A and B: Mounting diagnostic casts with full-mouth diagnostic wax-up and provisional restoration

relined in the patient's mouth and temporarily cemented on the prepared teeth. The patient was reevaluated after a period of time to assess her adaptation to the proposed new occlusal plane (Fig. 4B). Thereafter, maxillary and mandibular teeth preparations were finalized. Gingival retractions (Ultradent Products, Inc.) were implemented using a plain cord soaked in an aluminum chloride solution. The final impressions were obtained using the additional silicone polyvinyl siloxane impression material (Virtual, Ivoclar Vivadent, Lichtenstein) through double mixing technique (Figs $4 \mathrm{~A}$ to D).

In Phase III, centric relation records were made between the maxillary prepared teeth with opposing provisional restoration to allow cross-mounting of the casts using an anterior jig device. The casts were mounted on a Denar Mark II Articulator using an arbitrary face-bow and interocclusal registration material (EXABITE II NDS). The final impressions were poured with CAD/CAM improved special stone (BEGO, Germany). Dies were prepared, and the margins were ditched and marked. Two coats of die spacer were applied to each trimmed die (Figs $5 \mathrm{~A}$ and $\mathrm{B}$ ). Metals for copings were constructed using a laser scanner (Cynoprod, CANADA) of the CAD/ CAM machine for teeth \#17, 14, 11, 21, 23, and 26. The primary copings of the telescopic bridge in the maxillary arch were tried on and cemented on abutment teeth using the Rely $X^{\mathrm{TM}}$ Unicem Aplicap Resin Cement (3M ESPE, Germany). Bite registration and final impression were then done for the construction of telescopic maxillary FDP (Fig. 5C). A custom anterior guide table was fabricated using provisional casts. The mounted casts were sent to 

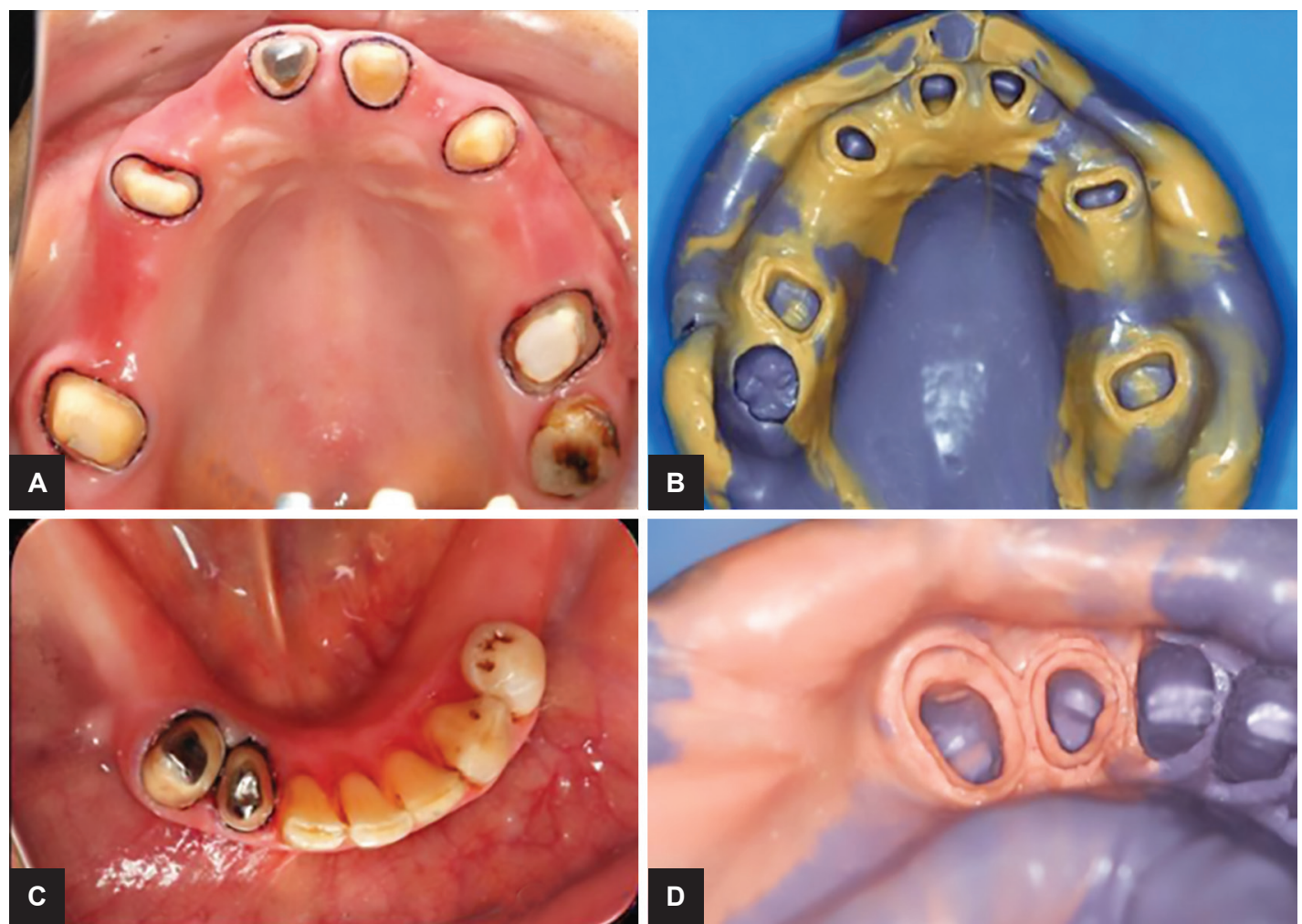

Figs 4A to D: Retraction cord application and final impression of both arches
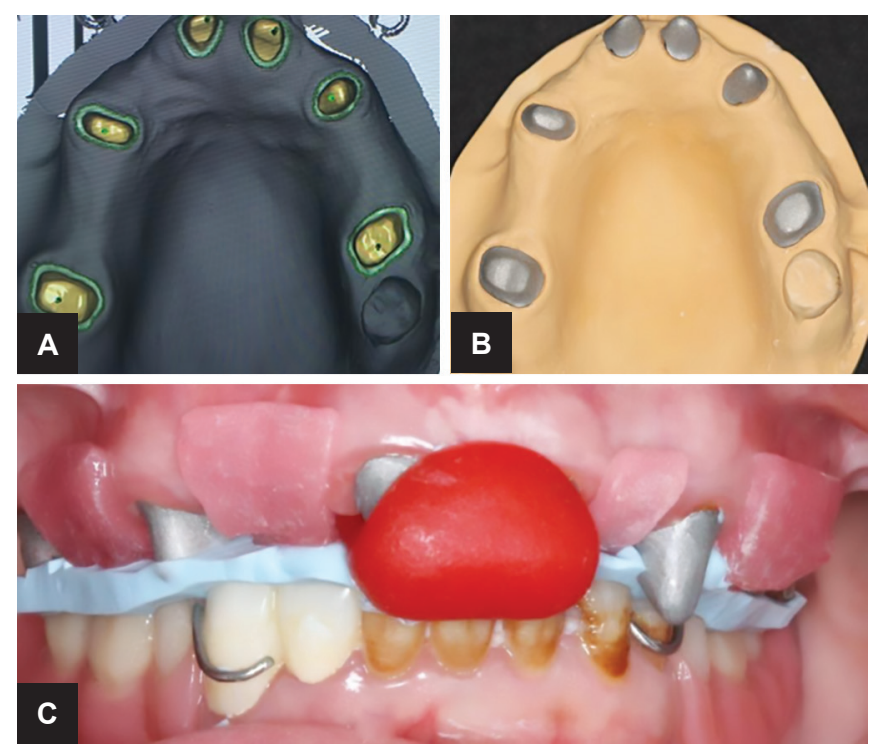

Figs 5A to C: Final cast after inner surface of TC cementation, die spacers application, and bite registration

the laboratory with the custom anterior guide table and a work authorization form. Subsequently, the secondary cores were milled off metals and the porcelain veneering was built up with Vita In-Ceram YZ Disk (VitaZahnfabric, Germany). Final MC FDPs were cemented on the existing copping using a temp-bond temporary luting agent (Temp-Bond NT, Italy; Fig. 6). Surveyed MC single crown on tooth \#44 and conventional crown on tooth \#43 with cobalt-chromium Kennedy class I RDP to replace the missing mandibular posterior teeth were fabricated and inserted according to the manufacturer's instructions. The fitting surfaces of the denture were inspected for sharp areas or excessive tissue undercuts that can cause irritation during insertion. The dentures were clinically remounted. The occlusion was refined on the articulator at the time of delivery. An occlusal view of the final prosthesis (Figs 7A and B) was obtained. Adjustments were made on all the prostheses inside the patient's mouth during the centric movement. The protrusive movement was guided by the natural existing centrals initially and then by anterior group function later in the movement. Posttreatment extraoral view during smile (Fig. 7C), panoramic and periapical radiographs were taken (Fig. 8) and Phase IV consisted mainly of follow-up visits after the insertion and cementation of the prosthesis. Oral hygiene was then reevaluated, and prosthesis adjustments and posttreatments were recorded. A periodic recall schedule was arranged for the patient after 48 hours, 1 week, and 3 weeks to examine the occlusion, irritation areas, esthetics, and overall comfort of the prostheses. Finally, the patient was placed on 6 months recall for reevaluation. Postoperative instructions were explained and reviewed with the patient including daily routine of thorough brushing technique, super flossing, proxy brushing, and fluoride application.

\section{DISCUSSION AND CLINICAL SIGNIFICANCE}

In the past 50 years, a considerable improvement has been achieved in material sciences for the restoration 

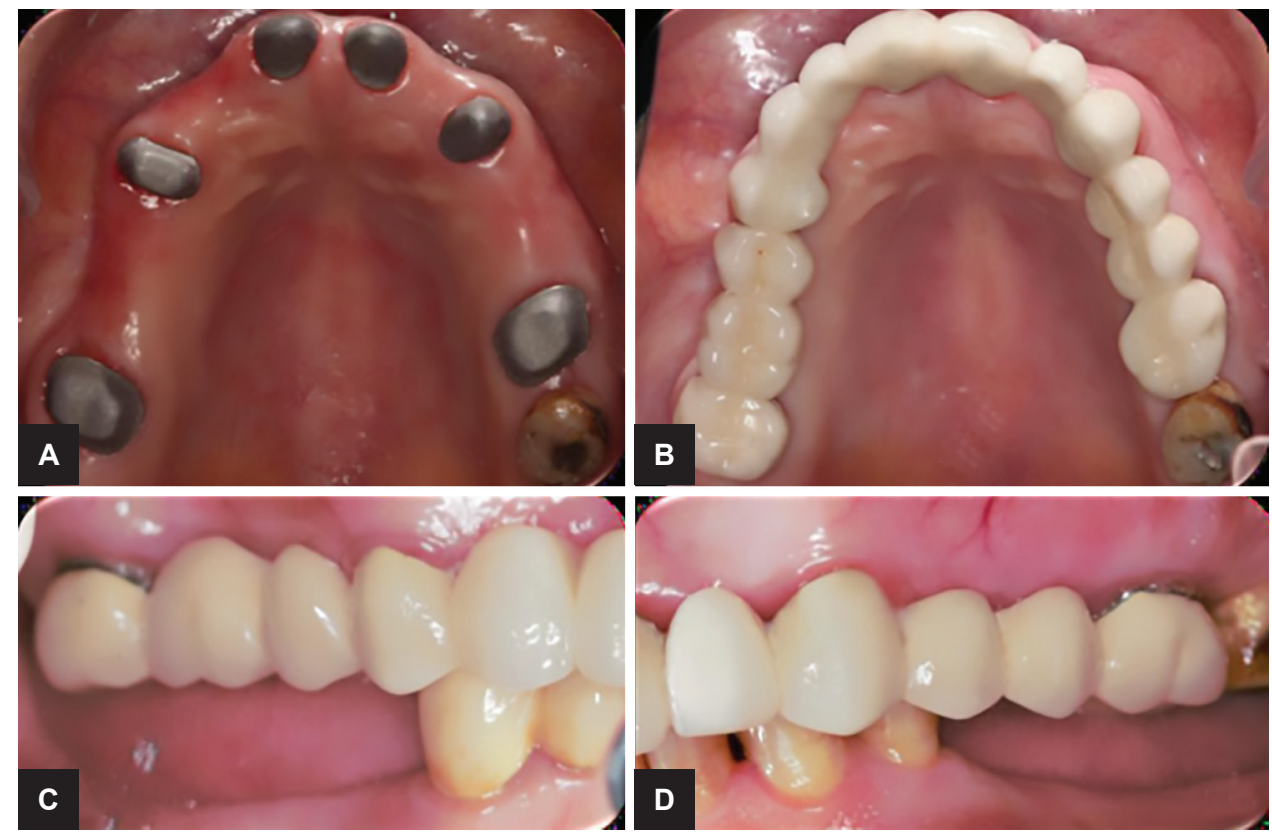

Figs 6A to D: Maxillary prosthesis seated in place (inner coping and outer coping of TC)
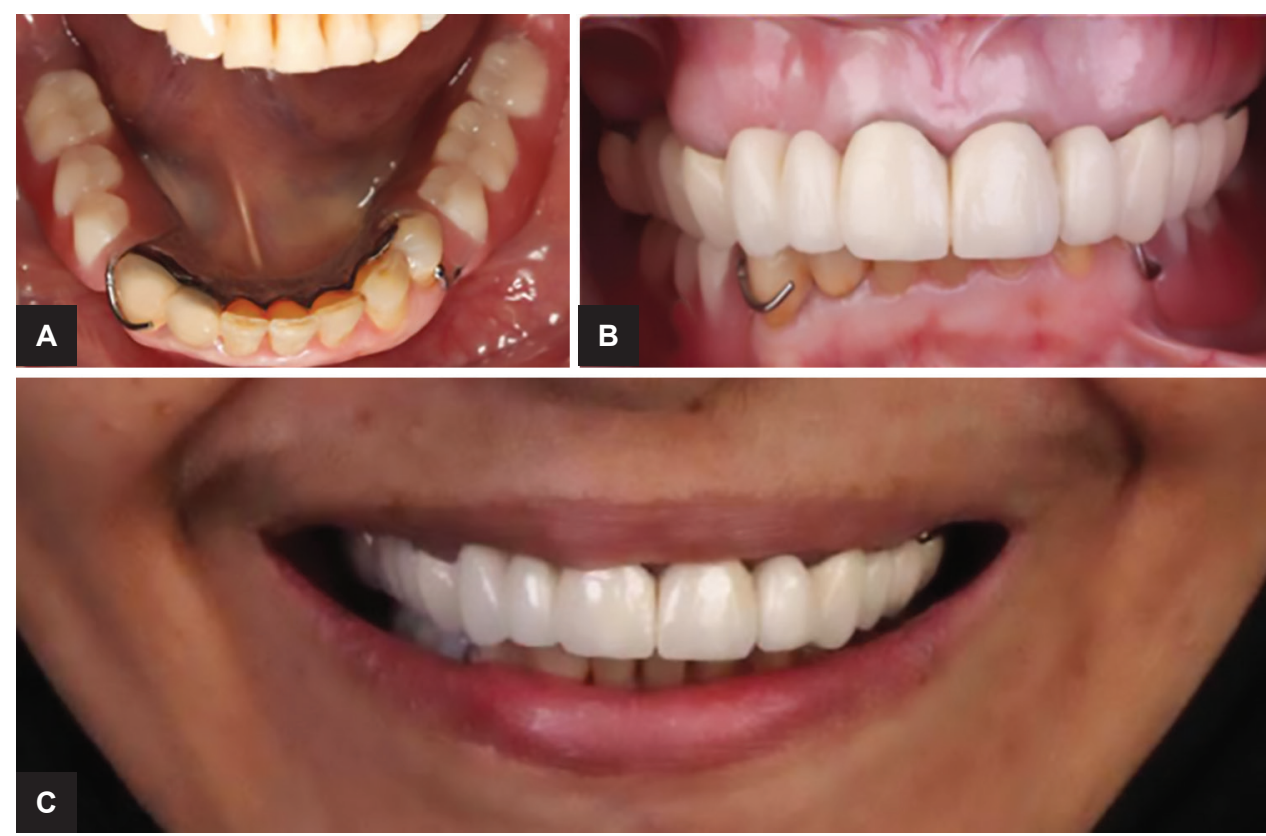

Figs 7A to C: Postoperative view with all prosthesis inside the mouth and extraoral postoperative view

of partially edentulous patients. Although often overlooked in the CAD/CAM technique and technology, telescopic retainers allow the use of prostheses that facilitate access for cleaning by the patient and/or the dentist and retain teeth with defects for a longer period of time..$^{10,11}$

The crown-root ratio, root configuration, and periodontal conditions are the main factors in the design and selection of abutment teeth for FDP. In the present case, all the factors satisfied the requirements given that the crown-root ratio of the existing teeth was 1:1 of the abutment, adequate root was embedded in the maxillary bone, and the periodontal ligament surface area was equal to or more than the surface area of the clinical crown. ${ }^{12}$

The FDPs with TCs are considerably costly compared with other treatment options, such as complete or partial overdenture and RDP; however, FDPs with TCs display many advantages over the RDP; these advantages include the preservation of all existing short clinical crowns or abutments, elimination of clasps used, satisfactory final esthetic, and preservation of all remaining natural teeth. ${ }^{13}$ In addition, the material used to veneer the TC is constructed from porcelain veneers; this material produces fewer bulks and excellent esthetic than 


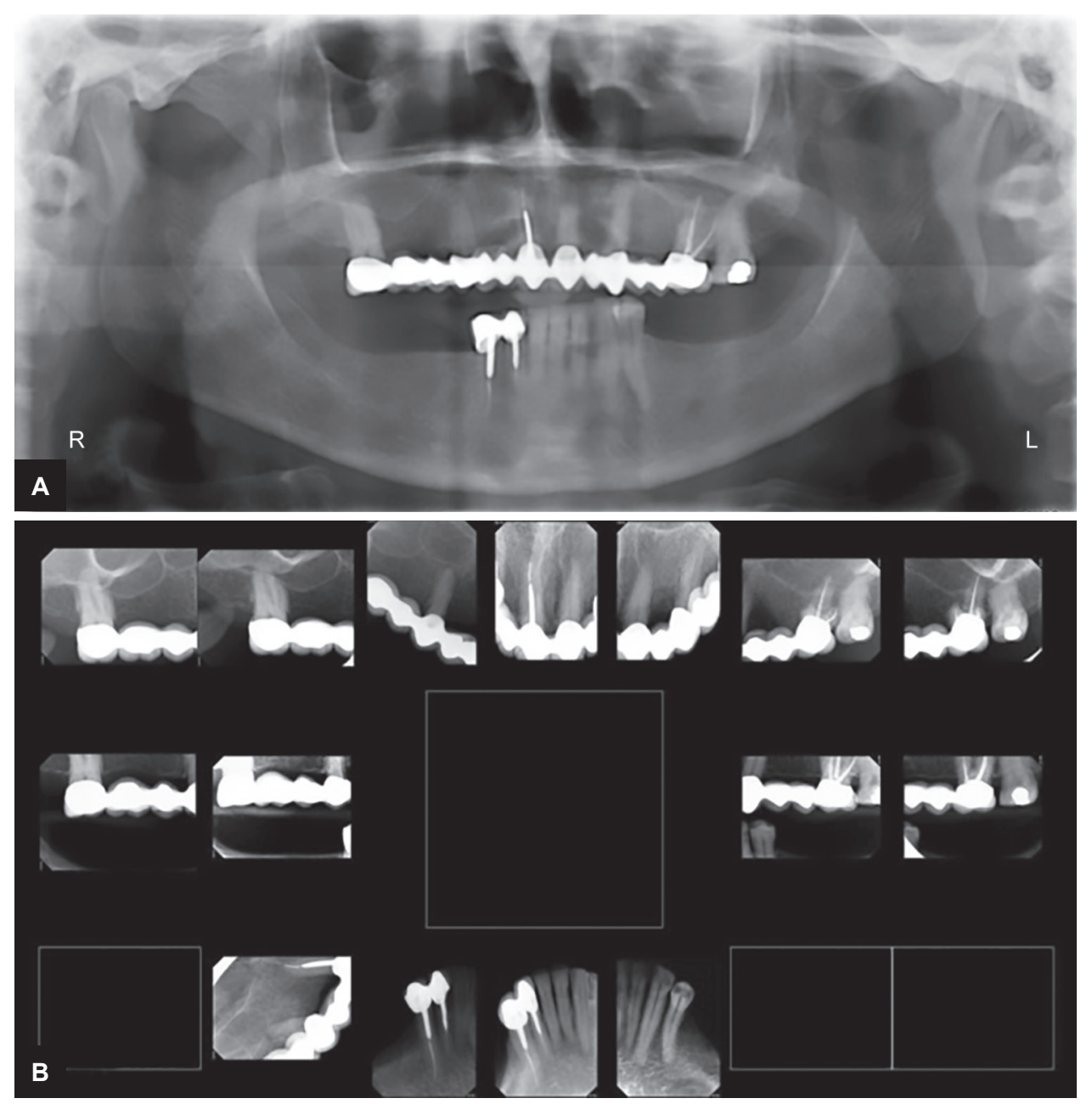

Figs $8 \mathrm{~A}$ and $\mathrm{B}$ : Postoperative radiographs

those of the TC in the past, which were constructed from acrylic facing. ${ }^{14}$ In this report, the TC in the maxillary arch was constructed with the aid of CAD/CAM technology, which designed with the maximum parallelism resulted in excellent retention of the FDP. Consequently, the adequate degrees of tapers, large surface areas, enough resistance, and retention of the prostheses during function were achieved. ${ }^{7}$

In this case, the primary coping of the TC was cemented using a resin cement, and the secondary coping of the telescopic FDP was cemented using a temporary cement. Given that the telescopic abutments in the maxillary FDP of the natural teeth may fail, the weak sealed cement between the metal-metal interfaces will fail first instead of debonding at the metal-tooth interfaces. ${ }^{11}$

Currently, dentists recognize that proper oral hygiene and the ability to maintain it are the most significant prophylaxes of periodontal disease and dental caries. ${ }^{7}$ This fact is evident in this current case during follow-up appointments. Patients and dentists should always consider that the most significant factor in maintaining the restoration is the ability to clean the abutments of the maxillary FDP and mandibular RDP with the surveyed crowns. ${ }^{7}$
The current case was followed up for 2 years. The result was consistent with that in a systematic review, which indicated that the survival rates of TC abutment teeth range from $82.5 \%$ after a 3.4 -year follow-up to $96.2 \%$ after a 6-year follow-up. ${ }^{15}$

A clinical significance of the presented case is that the secondary coping of the telescopic FDP is cemented with temporary cement, which can be changed easily after a period of function or whenever it is loose. This step is simple, inexpensive, and fast. Overall, the telescopic maxillary FDP is completely opposed to RDP in the mandibular posterior masticatory area. Consequently, the mastication forces are ineffective because the opposing teeth are artificial. Moreover, the short- and long-term prognoses of this case were good because the patient is medically fit and motivated. The patient developed an excellent, continual oral hygiene protocol. Finally, the patient developed a remarkably positive attitude and understood the importance of participating in the follow-up appointments.

\section{CONCLUSION}

Fixed dental prostheses supported with TCs are preferred in the rehabilitation of elderly partially edentulous 
patients over conventional RDPs because of several advantages including excellent esthetics, phonetics, stress distribution, retention, stability, stable occlusion, and chewing efficiency. The MC maxillary FDP may provide a low abrasive wear to the opposing natural teeth and acrylic teeth on the mandibular arch.

\section{REFERENCES}

1. Abraham PA, Koka P, Murugesan K, Vasanthakumar M. Telescopic overdenture supported by a combination of tooth and an implant: a clinical report. J Indian Prosthodont Soc 2010 Dec;10(4):230-233.

2. Langer $Y$, Langer A. Tooth-supported telescopic prostheses in compromised dentitions: a clinical report. J Prosth Dent 2000 Aug;34(2):129-132.

3. Shah N, Ram SN, Mhatre S, Mahadevan J. Telescopic overdenture with customized double copings-a simplified approach to preventive prosthodontics. J Cont Dent 2012 Jan-Apr;2(1):29-32.

4. Wenz HJ, Hertrampf K, Lehmann KM. Clinical longevity of removable partial dentures retained by telescopic crowns: outcome of the double crown with clearance fit. Int J Prosthodont 2001 May-Jun;14(3):207-213.

5. Cho JH, Cho SA. The use of telescopic crowns in removable partial denture treatments for patients with severe periodontal diseases: two patients with case history reports. Int J Prosthodont 2016 Mar-Apr;29(2):175-178.

6. Kumar L, Sehgal K. Removable partial denture supported by implants with prefabricated telescopic abutments-a case report. J Clin Diagn Res 2014 Jun;8(6):ZD04-ZD06.

7. Bergler M, Holst S, Blatz MB, Eitner S, Wichmann M. CAD/CAM and telescopic technology: design options for implant-supported overdentures. Eur J Esthet Dent 2008 Spring;3(1):66-88.

8. Singh K, Gupta N. Telescopic denture - a treatment modality for minimizing the conventional removable complete denture problems: a case report. J Clin Diagn Res 2012 Aug;6(6): 1112-1116.

9. Rosenstiel, SF.; Land, MF.; Fujimoto, J. Contemporary fixed prosthodontics. 4th ed. St. Louis (MO): Mosby Elsevier; 2006. pp. 174-187.

10. Alsayed HD, Alqahtani NM, Levon JA, Morton D. Prosthodontic rehabilitation of an ectodermal dysplasia patient with implant telescopic crown attachments. J Prosthodont 2017 Oct;26(7):622-627.

11. Breitman JB, Nakamura S, Freedman AL, Yaisove IL. Telescopic retainers: an old or new solution? A second chance to have normal dental function. J Prosthodont 2012 Jan;21(1): 79-83.

12. Shillinburg, HT Jr.; Sather, DA.; Wilson, EL Jr.; Cain, JR.; Mitchell, DL.; Blanco, LJ.; Kessler, JC. Fundamentals of fixed prosthodontics. 4th ed. Hanover Park (IL): Quintessence Publishing Co; 2012. p. 90-93, 119-120.

13. Dede DÖ, Durmuslar MC, Sahin O, Koroglu A, İşısağ Ö. Telescopic overdenture and implant supported fixed partial denture: a pragmatic treatment approach. Case Rep Dent 2015 Jun;2015:392-397.

14. Weigl P, Hahn L, Lauer HC. Advanced biomaterials used for a new telescopic retainer for removable dentures. J Biomed Mater Res 2000 Jul;53(4):320-336.

15. Verma R, Joda T, Brägger U, Wittneben JG. A systematic review of the clinical performance of tooth-retained and implantretained double crown prostheses with a follow-up of $\geq$ 3 years. J Prosthodont 2013 Jan;22(1):2-12. 